Mens

revue d'histoire intellectuelle de l'Amérique française

\title{
L'histoire intellectuelle en France
}

\section{Catherine Pomeyrols}

Volume 4, numéro 1, automne 2003

URI : https://id.erudit.org/iderudit/1024639ar

DOI : https://doi.org/10.7202/1024639ar

Aller au sommaire du numéro

Éditeur(s)

Centre de recherche en civilisation canadienne-française

ISSN

1492-8647 (imprimé)

1927-9299 (numérique)

Découvrir la revue

Citer ce document

Pomeyrols, C. (2003). L'histoire intellectuelle en France. Mens, 4(1), 159-176.

https://doi.org/10.7202/1024639ar d'utilisation que vous pouvez consulter en ligne.

https://apropos.erudit.org/fr/usagers/politique-dutilisation/ 


\author{
Bibliographie
}

\title{
L'HISTOIRE INTELLECTUELLE EN FRANCE
}

Catherine Pomeyrols

Université de Nantes

L'appellation " histoire intellectuelle » n'est plus utilisée en France, car elle apparaît désormais trop restreinte (les idées et leurs producteurs, les généalogies des courants de pensée) au regard de l'histoire des "mentalités » puis de celle des "pratiques culturelles» qui a remis l'accent sur les liens entre le culturel et le social. Cette histoire culturelle renouvelée s'est installée dans le paysage historiographique en liaison avec la remise en cause des paradigmes des Annales et des grands modèles explicatifs des années 1960 et 1970.

Il est désormais acquis, selon l'expression de Jacques Julliard dans un article des Annales en 1984, que les «idées ne se promènent pas toutes nues dans la rue " et qu'il faut, selon l'expression de Roger Chartier dans un article de cette même revue en 1989, penser "le monde comme représentation ». Cette histoire culturelle se veut globale, elle est conçue d'une façon très large et comprend à la fois l'histoire des idées et de leur diffusion, des institutions, des acteurs, des pratiques culturelles, des intellectuels, de l'éducation, des symboles, des arts, des loisirs, des croyances...

Elle s'est institutionnalisée dans les années 1980 (programme d'agrégation, séminaires et centres de recherche, édi- 
tion...), marquant le lien entre deux générations de chercheurs, l'effacement de la tradition d'histoire économique et sociale et la tentative de construction d'un modèle historiographique renouvelé. Héritière de l'histoire des idées, de l'anthropologie historique et de l'histoire des mentalités, elle a voulu rompre avec cet héritage.

Enfin, cette histoire culturelle française est associée à une histoire politique renouvelée après un long discrédit. En histoire contemporaine, René Rémond en fut à l'origine dans les années 1950 avec Les droites en France, incitant depuis de nouvelles générations d'historiens (Fondation nationale des sciences politiques, Université de Paris X Nanterre, Institut d'Histoire du Temps Présent...) à renouveler ce genre et à s'intéresser à des périodes plus proches dans le temps. Il faut également citer Maurice Agulhon, personnalité un peu en marge des " écoles », qui a aussi contribué à renouveler l'histoire politique et à faire advenir une histoire culturelle du contemporain en étudiant les sociabilités, les symboles et leur enracinement.

Michel Trebitsch (1990) repérait trois directions principales dans une histoire culturelle très hétérogène : une histoire socioculturelle (histoire sociale des représentations et des pratiques, développée par Roger Chartier, Alain Corbin...), une histoire culturelle du politique (Serge Berstein, René Rémond, Pascal Ory, Jean-François Sirinelli, Jean-Pierre Rioux...) et une histoire des symboles et des imaginaires sociaux (Maurice Agulhon, Pierre Nora et l'entreprise des Lieux de mémoire, Henri Rousso...).

Quelques années plus tard, dans l'introduction d'un ouvrage au titre volontairement programmatique, Jean-Pierre Rioux, dans Pour une bistoire culturelle, définit quatre " rivages sûrs » : l'histoire des politiques et des institutions culturelles (idéaux, acteurs, cultures politiques) ; l'histoire des médiations 
et des médiateurs (supports, circulation, objets, fêtes, loisirs, rites) ; l'histoire des pratiques culturelles (sociabilités, mémoires, identités); l'histoire des signes et des symboles.

Demeurée très diverse et pour certains parfois trop floue sur le plan conceptuel aux frontières de ces différents « rivages ", il n'est aujourd'hui pas certain que l'histoire culturelle constitue un domaine déterminé, même si elle en comprend en son sein, mais plutôt une extension du questionnaire de l'historien après les remises en question des années 1980, et selon l'expression de Philippe Urfalino, « le nom provisoire d'un vaste chantier de fouilles, ouvert par le déclin de grands modèles explicatifs et l'enrichissement de la boîte à outils de l'historien " (Vingtième siècle, $\mathrm{n}^{\circ} 57$, janvier-mars 1998).

On retrouvera dans cette bibliographie, dont l'exhaustivité n'est pas le but, les principales rubriques signalées ci-dessus : j’ai fait figurer en premier lieu un échantillon de l'historiographie portant sur cette thématique culturelle, en indiquant à la fois des ouvrages ayant fait date et quelques balises permettant de se repérer dans les évolutions récentes de l'historiographie. Viennent ensuite les principaux instruments de travail et quelques synthèses - dont je me suis efforcée d'indiquer les plus utiles et les plus récents - beaucoup de ces ouvrages comprenant bien sûr à leur tour des références bibliographiques où le chercheur ira puiser selon ses besoins. Les revues lui sont également indispensables, même si je n'ai indiqué que quelques articles ou numéros spéciaux, afin de se tenir au courant des débats, des tendances de la recherche et des parutions.

Sauf pour les outils de travail et éventuellement les grandes synthèses, cette première approche bibliographique, dans les thématiques indiquées, est limitée à la période contemporaine (XIX ${ }^{\mathrm{e}}-\mathrm{XX}^{\mathrm{e}}$ siècles) à l'exclusion de la Révolution française (1789-1799) et de la période napoléonienne (1799-1815). 
Ces ouvrages sont susceptibles d'intéresser des étudiants et les chercheurs dans une perspective comparatiste à la fois parce que l'histoire culturelle française est souvent très méconnue, hormis quelques domaines bien précis (le livre, l'édition, les bibliothèques), et parce que jusqu'à la Seconde Guerre mondiale, les élites intellectuelles québécoises y puisent encore beaucoup de leurs références. Tout étudiant ou chercheur désireux d'étudier l'histoire intellectuelle de la France devra en outre compléter cette base bibliographique par des lectures dans le champ « économique et social » - volontairement non traité ici —, les « idées » n'étant ni en apesanteur, ni des essences platoniciennes.

\section{Historiographie}

AGULHON Maurice, Histoire vagabonde, 3 tomes, Paris, Gallimard, 1988 et 1996.

BÉDARIDA François (dir.), L'bistoire et le métier d'bistorien 1945-1995, Paris, MSH, 1995.

BEAUVOIS Yves et BLONDEL Catherine (dir.), Qu'est-ce qu'on ne sait pas en histoire?, Lille, P. U. Septentrion, 1998.

BOUTIER Jean et JULIA Dominique (dir.), Passés recomposés, Paris, Autrement-Mutations, 1995.

BOURDIEU Pierre, Les règles de l'art. Genèse et structure du champ littéraire, Paris, Seuil, 1992 ; Seuil-Point-Essais 1998.

CERTEAU Michel de, L'écriture de l'histoire, Paris, GallimardNRF, 1975.

CHARTIER Roger, $A$ u bord de la falaise. L'bistoire entre certitudes et inquiétude, Paris, Albin Michel, 1998 [recueil d'articles essentiels dont «Le monde comme représentation » de 1989]. 
COQ Christian et BACOT Jean-Pierre, Travail de mémoire, 1914-1998 : une nécessité dans un siècle de violence, Paris, Autrement, 1999.

DOSSE François, L'bistoire en miettes. Des Annales à la Nouvelle histoire, Paris, La Découverte, 1987.

Écrire l'bistoire du temps présent, Paris, CNRS / IHTP, 1993.

ESPAGNE Michel, "Sur les limites du comparatisme en histoire culturelle ", Genèses, 17, septembre 1994, pp. 112-21.

FOUCAULT Michel, L'archéologie du savoir, Paris, Gallimard, 1969.

KOSSELECK Reinhart, L'expérience de l'bistoire, Paris, SeuilGallimard, 1997.

KOSSELECK Reinhart, Le futur passé. Contribution à la sémantique des temps historiques, Paris, EHESS, 1990.

L'bistoire aujourd'bui. Nouveaux objets de recherche. Courants et débats, le métier d'historien, Paris, Éditions sciences humaines, 1999.

Le Débat, $\mathrm{n}^{\circ} 103$, janvier-février 1999, "Inquiétudes et certitudes de l'histoire $»$.

LE GOFF Jacques et NORA Pierre (dir.), Faire de l'histoire, Paris, Gallimard, 1974. rééd. 1986 [la " nouvelle histoire », héritière des Annales].

NOIRIEL Gérard, " L'histoire culturelle aujourd'hui. Entretien avec Roger Chartier. ", Genèses, 15, mars 1994, pp. 115-29.

PROST Antoine, Douze leçons sur l'histoire, Paris, Seuil PointHistoire, 1996 [excellente mise au point, importante bibliographie sur l'historiographie]. 
RICCEUR Paul, Temps et récit, Paris, Seuil, 1983-1985 ; rééd. Seuil-Point-Histoire 1991.

RICCEUR Paul, La mémoire, l'Histoire, l'Oubli, Paris, Seuil, 2000 ; rééd. Seuil-Point-Essais 2003.

RIOUX Jean-Pierre, SIRINELLI Jean-François, Pour une bistoire culturelle, Paris, Seuil, 1997.

TREBITSCH Michel, «Promesses et problèmes de l'histoire culturelle », dans Premières recherches, Paris, Histoire au présent/ Boutique de l'Histoire/Publications de la Sorbonne, 1990, pp. 151-56.

\section{Instruments de travail}

BELLANGER Claude, CHARLET Louis et GODECHOT Jacques, Histoire générale de la presse française, Paris, PUF, 19721976.

BERSTEIN Serge et Gisèle, Dictionnaire historique de la France contemporaine, 1870-1945, Bruxelles, Complexe, 1995.

BURGUIÈRE André (dir.), Dictionnaire des sciences historiques, Paris, PUF, 1986.

CHARLE Christophe, Les professeurs de la faculté des lettres de Paris. Dictionnaire biographique (1909-1939), Paris, INRP / CNRS, 1986.

CHATELET François, DUHAMEL Olivier, PISIER Evelyne, Dictionnaire des xuvres politiques, Paris, PUF, $3^{e}$ édition, 1995.

CHAUVEAU Agnès et JEANNENEY Jean-Noël, L'écho $d u$ siècle. Dictionnaire historique de la radio et de la télévision en France, [s.l.], Hachette-ARTE, 1999. 
DE WARESQUIEL Emmanuel (dir.), Le siècle rebelle. Dictionnaire de la contestation au XXe siècle, Paris, Larousse, 1999. DUCLERT Vincent et PROCHASSON Christophe (dir.), Dictionnaire critique de la République, Paris, Flammarion, 2002. FURET François et OZOUF Mona, Dictionnaire critique de la Révolution française, Paris, Flammarion, 1988.

JOLY Bertrand, Dictionnaire biographique et géographique du nationalisme français (1880-1900). Boulangisme, Ligue des Patriotes, mouvements antidreyfusards, comités antisémites, Paris, Champion, 1997.

JULLIARD Jacques, WINOCK Michel (dir.), Dictionnaire des intellectuels français. Les personnes, les lieux, les moments, Paris, Seuil, 1996.

LAGUERRE Bernard, "Orientation bibliographique. Les générations d'intellectuels dans la France du $\mathrm{XX}^{\mathrm{e}}$ siècle ", dans Bulletin de l'IHTP, $\mathrm{n}^{\circ}$ 31, mars 1988, pp. 23-43.

LE QUILLEC Robert, La Commune de Paris, bibliographie critique, Paris, La Boutique de l'Histoire, 1997.

LIGOU Daniel, Dictionnaire de la franc-maşonnerie et des francsmaçons, Paris, PUF, 3e édition 1998.

Les idées en France, 1945-1988. Une chronologie, Paris, GallimardLe Débat, 1989.

MAITRON Jean (dir.), Dictionnaire biographique du mouvement ouvrier français, Paris, Éditions ouvrières, 1964-93 [4 séries : 1789-1864, 1864-1871, 1871-1914, 1914-1939 ; Parti socialiste, Parti communiste, anarchistes, militants, syndicalistes, politiques, théoriciens...]; Volume 44 Biographies nouvelles : 1789-1939, Paris, Éditions de l'Atelier, 1997. 
POULOT Dominique, Bibliographie de l'bistoire des musées de France, Paris, CTHS, 1994.

Prosopographie des élites françaises (XVT $-X X^{e}$ siècles). Guide de recherche, Paris, IHMC / CNRS, 1980.

Revue d'Histoire Moderne et Contemporaine, janvier-mars 1992, $\mathrm{n}^{\circ}$ spécial "Pour une histoire culturelle du contemporain ».

RIOUX Jean-Pierre (dir.), Histoire culturelle de la France contemporaine. Bilans et perspectives de recherche, Paris, Ministère de la culture-CNRS, 1987.

RIOUX Jean-Pierre et SIRINELLI Jean-François (dir.), La France d'un siècle à l'autre 1914-2000. Dictionnaire critique, Paris, Hachette, 1999.

SIRINELLI Jean-François (dir.), Dictionnaire historique de la vie politique francaise an XX siècle, Paris, PUF, 1995.

SOBOUL Albert et SURRATEAU Jean-René, Dictionnaire bistorique de la Révolution française, Paris, PUF, 1989.

TULARD Jean (dir.), Dictionnaire Napoléon, Paris, Fayard, 1987.

TULARD Jean (dir.), Dictionnaire du Second Empire, Paris, Fayard, 1995.

\section{Synthèses générales}

LEQUIN Yves, Histoire des Français, $X I X^{e}-X X^{e}$ siècles, 3 volumes, Paris, Colin, 1984.

NORA Pierre (dir.), Les lieux de mémoire, 3 tomes, 7 volumes : La république, La Nation, Les France, Paris, GallimardBibliothèque des histoires, 1984, 1986, 1992 ; rééd. Gallimard-Quarto, 1997. 
Nouvelle bistoire de la France contemporaine (NHFC), Paris, SeuilPoint-Histoire, 20 tomes dont le dernier de documents.

ZELDIN Théodore, Histoire des passions françaises 1848-1945, 2 tomes, Paris, Payot-Rivages, rééd. 1994.

\section{Synthèse histoire culturelle}

BURGUIÈRE André et REVEL Jacques, Histoire de la France, Paris, rééd. Seuil-Point-Histoire 2000, 5 volumes : Les conflits, Choix culturels et mémoire, Héritages, L'espace français, La longue durée de l'État.

CORBIN Alain, GEORGEL Chantal et al, L'invention du XIX siècle. Le XIX' siècle par lui-même (littérature, bistoire, société), Paris, Klincksieck, 1999.

DONNAT Olivier et COGNEAU Denis, Les pratiques culturelles des Français 1973-1989, Paris, La Découverte, 1990.

GOETSCHEL Pascale et LOYER Emmanuelle, Histoire culturelle et intellectuelle de la France an $X X^{e}$ siècle, Paris, ColinCursus, 1995.

Histoire culturelle de la France, Paris, Seuil, 1998 : DE BÆCQUE Antoine et MELONIO François (dir.), tome 3 : Lumières et liberté. Les XVIII' et XIX' siècles ; RIOUX Jean-Pierre et SIRINELLI Jean-François, tome $4:$ Le temps des masses. Le XX ${ }^{c}$ siècle.

L'aventure intellectuelle de la France au $X X^{*}$ siècle, Thierry PAQUOT (dir.), Paris, La Découverte; 3 parus: PROCHASSON Christophe, Les années électriques (18801910), 1991 ; LINDENBERG Daniel, Les années souterraines, (1937-1947), 1990 ; DELANNOI Gil, Les années utopiques (1968-1978), 1990. 
ORY Pascal, L'aventure culturelle française, Paris, Flammarion, 1989.

POIRRIER Philippe, Société et culture en France depuis 1945, Paris, Seuil-Mémo, 1998.

\section{Principales revues à dépouiller}

Annales historiques de la Révolution française, Annales Histoire Sciences Sociales, Actes de la Recherche en Sciences Sociales, Bulletin de I'IHTP (Institut d'Histoire du Temps Présent-CNRS), Esprit, Genèses, Histoire de l'éducation, Historiens et géographes* [bibliographies très complètes des quatre questions mises au concours de l'Agrégation d'histoire, elles changent par deux tous les deux ans, parution des bibliographies dans le numéro de l'été], Le Débat, Le Monvement social, Mil Neuf Cent-revue d'bistoire intellectuelle, Revue d'Histoire du XIX'e siecle, Revue des revues, Revue bistorique, Revue d'Histoire Moderne et Contemporaine, Sciences bumaines, Vingtième Siècle.

* Voir en particulier la question d'Agrégation 1988-1989 qui fit date : " Histoire culturelle de l'Europe occidentale de 1919 à la fin des années cinquante : croyances et pratiques religieuses, idées et pratiques politiques, courants intellectuels et artistiques et leurs relations avec les mentalités collectives »; bibliographie publiée dans le numéro 315 d'octobre 1987.

\section{Bibliothèques-Édition-Lecture-Médias}

BARBIER Frédéric et BERTHO-LAVENIR Catherine, Histoire des médias de Diderot à Internet, Paris, A. Colin-U, 1996. BERTHO-LAVENIR Catherine, La démocratie et les médias de 1918 à nos jours, Paris, Colin-U, 1999. 
BODEKER Hans-Erich (dir.), Histoires du livre. Nouvelles orientations, Paris, IMEC-MSH, 1995.

BROCHAND Christian (dir.), Histoire de la radio et de la télévision en France, Paris, La documentation française, 1994.

CHARTIER Roger (dir.), Histoires de la lecture. Un bilan des recherches, Paris, IMEC-MSH, 1995.

CHARTIER Roger et MARTIN Henri-Jean (dir.), Histoire de l'edition française, tome II : 1660-1830, tome III : 1830-1900, tome IV : 1900-1950, Paris, Fayard, 1990-1991.

DELPORTE Christian, Les journalistes en France, 1880-1950. Naissance et construction d'une profession, Paris, Seuil-Univers Historique, 1999.

Histoire des bibliothèques francaises: VARRY Dominique, tome III : 1789-1914, Paris, Promodis, 1991 ; POULAIN Martine, tome IV : 1914-1990, Paris, Promodis, 1992.

MOLLIER Jean-Yves (dir.), Le commerce de la librairie en France an XIX', Paris, IMEC-MSH, 1997.

MOLLIER Jean-Yves et SOREL Patricia, "L'histoire de l'édition, du livre et de la lecture en France aux XIX ${ }^{\mathrm{e}}$ et $\mathrm{XX}^{\mathrm{e}}$ siècles. Approche bibliographique ", Actes de la recherche en sciences sociales, $\mathrm{n}^{\circ \mathrm{s}}$ 126-127, mars 1999, pp. 39-59.

MOLLIER Jean-Yves, La lecture et ses publics à l'époque contemporaine. Essais d'bistoire culturelle, Paris, PUF, 2001.

\section{Religion}

BAUBÉROT Jean, Histoire de la laüité française, Paris, PUFQue Sais-Je?, 2000.

BENBASSA Esther, Histoire des Juifs de France, Paris, Seuil, 1997. 
CARBONNIER-BURKARD Marianne et CABANEL Patrick, Une histoire des protestants en France, Paris, Desclée de Brouwer, 1998.

CHOLVY Gérard et HILAIRE Yves-Marie (dir.), Histoire religieuse de la France contemporaine, Toulouse, Privat, Tome 1 : 1800-1880, 1985 ; Tome 2 : 1880-1930, 1986 ; Tome 3 : 19301988, 1988.

COLIN Pierre et FOUILLOUX Étienne (dir.), Intellectuels chrétiens et esprit des années vingt, Paris, Cerf, 1997.

FOUILLOUX Étienne, " "Intellectuels catholiques" ? Réflexions sur une naissance différée ", Vingtième siècle, 53, janvier-mars 1997, pp. 13-24.

FOUILLOUX Étienne, Une Église en quête de liberté. La pensée catholique française entre modernité et Vatican II (1914-1962), Paris, Desclée de Brouwer, 1998.

Historiens et Géographes, $\mathrm{n}^{\circ} 375$, juillet-août 2001. Bibliographie de la question d'Agrégation "Religion et culture dans les sociétés et les états européens de 1800 à 1914 (Allemagne, France, Italie, Royaume-Uni) ».

LE GOFF Jacques et RÉMOND René (dir), Histoire de la France religieuse, 4 tomes, Paris, PUF, 1992.

LEBRUN François (dir.), Histoire des catholiques en France, Paris, Hachette - Pluriel, 1985.

MAYEUR Jean-Marie, PIÉTRI Charles, VAUCHEZ André et VENARD Marc (dir.), Histoire du christianisme. Tome XI : Libéralisme, industrialisation, expansion européenne (1830-1914), tome XII : Guerres mondiales et totalitarismes (1914-1958), Paris, Desclée/Fayard, 1990 et 1995.

PRÉVOTAT Jacques, Les catboliques et l'Action française. Histoire d'une condamnation 1899-1939, Paris, Fayard, 2001. 
RÉMOND René, "L'histoire des intellectuels catholiques. Interview par Jacques Julliard et Daniel Lindenberg ", Mil Neuf Cent, 13, 1995, pp. 14-24.

\section{Enseignement}

BOURDIEU Pierre et PASSERON Jean-Claude, Les Héritiers. Les étudiants et la culture, Paris, Minuit, 1964 et La Reproduction. Éléménts pour une théorie du système d'enseignement, Paris, Minuit, 1970.

CHANET Jean-François, L'école républicaine et les petites patries, Paris, Aubier-Histoires, 1996.

CHALINE Nadine-Josette, CHOLVY Gérard, L'enseignement catholique en France aux XIX et XX $X^{e}$ siècles, Paris, Cerf, 1995.

CRUBELLIER Maurice, L'école républicaine, 1870-1940. Esquisse d'une histoire culturelle, Paris, Éditions Christian, 1993.

Histoire générale de l'enseignement et de l'éducation, Paris, Nouvelle Librairie de France, 1981, 4 volumes.

PROST Antoine, Histoire de l'enseignement en France, 1800-1967, Paris, A. Colin, 1968.

PROST Antoine, Éducation société et politique. Une histoire de l'enseignement de 1945 à nos jours, Paris, Seuil-Point-Histoire, 1997.

VERGER Jacques (dir.), Histoire des universités en France, Toulouse, Privat, 1986.

\section{Idéologies - Politique}

AGULHON Maurice, Marianne au combat : limagerie et la symbolique républicaine de 1789 à 1880 ; Marianne au pouvoir (1880-1914) ; Les métamorphoses de Marianne (1914 à nos jours), Paris, Flammarion, 1979-1989-2001. 
AGULHON Maurice (dir.), Cultures et folklores républicains, Paris, CTHS, 1995.

ANDRIEU Claire, LE BEGUEC Gilles et TARTAKOWSKY Danielle (dir.), Associations et champ politique. La loi de 1901 à l'épreuve du siècle, Paris, Publications de la Sorbonne, 2001.

AUDOIN-ROUZEAU et al (dir.), La politique et la guerre. Pour comprendre le XX' siècle, Paris, Noesis, 2002.

BECKER Jean-Jacques et al, Guerres et cultures. 1914-1918, Paris, A. Colin, 1994.

BERSTEIN Serge (dir.), Les cultures politiques en France, Paris, Seuil, 1999, rééd. Seuil-Point-Histoire, 2003.

BUTON Philippe, Une histoire intellectuelle de la démocratie, 19181989, Paris, Seli Arslan, 2000.

BURRIN Philippe, La dérive fasciste. Doriot, Déat, Bergery. $1933-$ 1945, Paris, Seuil, 1986.

CHARLE Christophe, La République des universitaires, 1870 1940, Paris, Seuil, 1994.

CHEVALLIER Pierre, Histoire de la franc-maçonnerie française, Paris, Fayard, 1973-1975.

CCEURÉ Sophie, La grande lueur à l'Est. Les Français et l'Union soviétique 1917-1939, Paris, Seuil, 1999.

CORBIN Alain, GÉRÔME Nicolas et TARTAKOWSKY Danièle (dir.), Les usages politiques des fêtes aux XIX ${ }^{e}$ et $X X^{e}$ siècles, Paris, Publications de la Sorbonne, 1994.

DELANNOI Gil et TAGUIEFF Pierre-André, Théories $d u$ nationalisme, Paris, Kimé, 1991.

DROZ Jacques (dir.), Histoire générale du socialisme, Paris, rééd. PUF-Quadrige, 1997. 
GÉRARD Alice, La Révolution française, mythes et interprétations, Paris, Flammarion, 1970.

LALOUETTE Jacqueline, La libre pensée en France, 1848-1940, Paris, Albin Michel, 1997.

LEBOVICS Herman, La vraie France, les enjeux de lidentité culturelle, 1900-1945, Paris, Belin, 1996.

LEVEQUE Pierre, Histoire des forces politiques en France, 3 tomes (de 1789 à nos jours), Paris, A. Colin, 1992-1997.

MILZA Pierre, Fascisme français. Passé et présent, Paris, Flammarion, 1987.

NGUYEN Victor, Aux origines de l'Action Française. Intelligence et politique à l'aube du XX' siècle, Paris, Fayard, 1991.

NICOLET Claude, L'idée républicaine en France, essai d'bistoire critique, Paris, Gallimard, 1982.

ORY Pascal (dir.), Nouvelle histoire des idées politiques, Paris, Hachette, 1987.

RÉMOND René (dir.), Pour une histoire politique, Paris, Seuil, 1988.

RÉMOND René, Les droites en France, Paris, Aubier Montaigne, 1982.

ROSANVALLON Pierre, Le sacre du citoyen. Histoire du suffrage universel en France, Paris, Gallimard, 1992.

ROUSSO Henri, Le syndrome de Vichy de 1944 à nos jours, Paris, Seuil, 1987, rééd. Seuil-Point-Histoire, 1990.

SADOUN Marc (dir.), La démocratie en France, tome 1 : Idéologies, tome 2 : Limites, Paris, Gallimard, 2000. 
SIRINELLI Jean-François (dir.), Histoire des droites en France, Paris, Gallimard, 1992 ; trois volumes : Politique, Cultures, Sensibilités.

STERNHELL Zeev, La droite révolutionnaire. Les origines françaises du fascisme, 1885-1914, Paris, Seuil, 1978.

TARTAKOWSKI Danielle, Les manifestations de rue en France: 1918-1968, Paris, Publications de la Sorbonne, 1998.

WINOCK Michel, La gauche en France depuis 1789, Paris, SeuilPoint-Histoire, 1995.

\section{Intellectuels}

BALMAND Pascal, "L'anti-intellectualisme dans la culture politique française ", Vingtième siècle, $\mathrm{n}^{\circ} 36$, octobre-décembre 1992, pp. 31-42.

BOURDIEU Pierre, Homo academicus, Paris, Minuit, 1984.

BOURDIEU Pierre, La noblesse d'État, Paris, Minuit, 1989 [sur les grandes écoles].

Cabiers de l'IHTP, $\mathrm{n}^{\circ}$ 6, novembre 1987, SIRINELLI JeanFrançois (dir.), Générations intellectuelles.

Cabiers de l'IHTP, $\mathrm{n}^{\circ}$ 20, mars 1992, RACINE Nicole et TREBITSCH Michel (dir.), Sociabilités intellectuelles. Lieux, milieux, réseaux.

CHALINE Jean-Pierre, Sociabilité et érudition. Les sociétés savantes en France $X I X^{e}-X X^{e}$ siècles, Paris, CTHS, 1995 (éd. poche, 1998).

CHARLE Christophe, Naissance des "intellectuels " 1880-1900, Paris, Éditions de Minuit, 1990.

CHARLE Christophe, Les intellectuels en Europe au XIX siècle. Essai d'histoire comparée, Paris, Seuil, 1996. 
CHARTIER Roger (dir.), La correspondance. Les usages de la lettre au XIX siècle, Paris, Fayard, 1991.

DUJARDIN Philippe, Du groupe au réseau. réseaux religieux, politiques, professionnels, Paris, CNRS, 1988.

GISPERT Hélène (dir.), "Par la science, pour la patrie ». L'Association Francaise pour l'Avancement des Sciences (18721914), un projet politique pour une société savante, Rennes, PUR, 2002.

GRANJON Marie-Christine, TREBITSCH Michel (dir.), Pour une histoire comparée des intellectuels, Bruxelles, Complexe-IHTP, 1998.

LOUBET DEL BAYLE Jean-Louis, Les non-conformistes des années trente, Paris, Seuil, 1969.

ORY Pascal (dir.), Dernières questions aux intellectuels, Paris, O. Orban, 1990.

ORY Pascal et SIRINELLI Jean-François, Les intellectuels en France, de l'affaire Dreyfus à nos jours, Paris, A. Colin, 1986, $3^{\mathrm{e}}$ édition 2002.

PROCHASSON Christophe, Les intellectuels et le socialisme, Paris, Plon, 1997.

RIEFFEL Rémy, La tribu des clercs. Les intellectuels sous la $V^{e}$ République, Paris, Calmann-Lévy, 1993.

SIRINELLI Jean-François, Génération intellectuelle. Khâgneux et normaliens dans l'entre-deux-guerres, Paris, Fayard, 1988.

\section{Politique culturelle - Loisirs}

Cabiers de l'IHTP, $\mathrm{n}^{\circ}$ 16, septembre 1990, RIOUX Jean-Pierre et SIRINELLI Jean-François (dir.), Les politiques culturelles municipales. Éléments pour une approche historique. 
CORBIN Alain, L'avènement des loisirs 1850-1960, Paris, Aubier, 1995.

DUBOIS Vincent, La politique culturelle. Genèse d'une catégorie d'intervention publique, Paris, Champion, 1999.

KALIFA Dominique, La culture de masse en France. Paris, La découverte, 2001, tome 1: 1860-1930.

ORY Pascal, La belle illusion. Culture et politique sous le signe du Front populaire, 1935-1938, Paris, Plon, 1994.

POIRRIER Philippe, Histoire des politiques culturelles de la France contemporaine, Dijon, PUD, 1996.

POIRRIER Philippe, Bibliographie de l'bistoire des politiques culturelles. France $X I X^{e}-X X^{e}$, Paris, Comité d'histoire du Ministère de la culture, 1999.

RIOUX Jean-Pierre et SIRINELLI Jean-François (dir.), Histoire des politiques et des institutions culturelles en France des années 1940 à nos jours, Paris, IHTP-CNRS, 1990.

RIOUX Jean-Pierre et SIRINELLI Jean-François, La culture de masse en France de la Belle Époque à aujourd'bui, Paris, Fayard, 2002.

URFALINO Philippe, L'invention de la politique culturelle, Paris, La Documentation française, 1996.

YONNET Paul, Jeux, modes et masses, Paris, Minuit, 1985. 